\title{
OAB: Retardkapsel für mehr Therapietreue
}

- Einen deutlichen Fortschritt bei der medikamentösen Behandlung der Volkskrankheit überaktive Blase verspricht eine neu eingeführte Retardformulierung des Anticholinergikums Trospiumchlorid (Trospium XR $60 \mathrm{mg}$, uriVesc ${ }^{\circledR}$ ). Insbesondere den Bedürfnissen der überwiegend älteren, oft multimorbiden Patienten kommt die lediglich einmal tägliche Einnahme entgegen. Außerdem konnte die Verträglichkeit des antimuskarinen Wirkstoffs mit der neuen galenischen Form weiter verbessert werden. Trospiumchlorid ist das einzige quartäre Amin unter den bei überaktiver Blase eingesetzten Anticholinergika. Es ist hydro- phil und überschreitet im Gegensatz zu lipophileren Präparaten nur in sehr geringem Maße die Blut-Hirn-Schranke, informierte Dr. Christian Gratzke, München. Kognitive Einschränkungen sind deshalb kaum zu erwarten. Fast um die Hälfte seltener führte das Retardpräparat laut Gratzke in klinischen Studien zu der gefürchteten Nebenwirkung Mundtrockenheit (10,7\%). Da der Wirkstoff zum überwiegenden Teil direkt durch die Niere ausgeschieden und nicht in der Leber metabolisiert wird, sind Interaktionen mit anderen Medikamenten ebenfalls selten. Die therapeutische Effizienz wird zudem durch lokale Effekte im
Urogenitaltrakt erhöht. Auch bei adipösen Patienten, einer Risikogruppe für Inkontinenzprobleme, führte die neue Retardkapsel laut Prof. Helmut Madersbacher, Innsbruck, zu guten Ergebnissen.

Grundsätzlich sollte die medikamentöse Therapie bei überaktiver Blase nach sorgfältiger Abklärung in ein interdispziplinäres Therapiekonzept eingebunden sein, betonte Madersbacher. Beckenbodengymnastik sowie eine Verhaltenstherapie können wesentlich zum Therapieerfolg beitragen.

Pressekonferenz „Trospiumchlorid: Neue Option bei der 1X1-Behandlung der überaktiven Blase“, Mannheim, 14. November 2009; Veranstalter: Madaus

\section{Vorzeitiger Samenerguss: SSRI verlängert Zeit bis zur Ejakulation signifikant}

— Die mediane intravaginale EjakulationsLatenzzeit (IELT) liegt bei 5,4 Minuten. „Das sollte der beratende Arzt wissen, wenn ein Mann wegen vermeintlich vorzeitigem Samenerguss in die Praxis kommt“, erklärte Prof. Dr. Hartmut Porst aus Hamburg. Denn möglicherweise ist einfach dessen Erwartungshaltung zu hoch. Bei der echten Ejaculatio praecox (EP), von der jeder vierte bis fünfte Mann unabhängig vom Alter betroffen ist, ist die IELT mit weniger als zwei Minuten deutlich reduziert und der Samenerguss kann nicht kontrolliert werden. 90\% dieser Männer „kommen“ bereits innerhalb der ersten 60 Sekunden. Die Folge ist ein erheblicher persönlicher Leidensdruck für Betroffene und Partner: Orgasmusprobleme etwa kommen bei Partnerinnen von Männern mit EP mehr als doppelt so häufig vor. Bei der schwersten Form, der Ejaculatio ante portas, findet der Samenerguss schon vor der Penetration statt - hier ist unerfüllter Kinderwunsch eine häufige zusätzliche Komplikation.

Selbst praktizierte Behandlungen - von Ablenkung und Unterbrechung der Stimulation, über Masturbation bis zu Alkohol und Drogen - führten ebenso wie Sexualtherapie nicht zum Erfolg, denn die EP sei zu 90\% mit einer Veränderung im Serotonintransportsystem genetisch determiniert, führte Porst weiter aus. Selten ist die EP erworben und nach vormals normaler Ejakulation etwa durch Erektionsstörungen, Prostatitis oder Schilddrüsenerkrankungen ausgelöst. Behandlungsversuche wurden in der Vergangenheit auch mit selektiven Serotoninwiederaufnahmehemmern (SSRI) oder trizyklischen Antidepressiva unternommen, waren aber Off label.

Mit Dapoxetin (Priligy ${ }^{\circledR}$ ) ist seit 2009 ein SSRI für die Therapie der EP zugelassen, der speziell für dieses Leiden entwickelt worden ist. Der Wirkstoff erhöht die Serotoninkonzentration im synaptischen Spalt und am postsynaptischen Rezeptor. Rascher
Wirkeintritt und schnelle Elimination ermöglichen eine Behandlung nach Bedarf. In Studien verlängerte das Medikament die IELT um das Drei- bis Vierfache und verbesserte die Zufriedenheit mit dem Geschlechtsverkehr bei Betroffenen und Partnern.

Aus der urologischen Praxis berichtete Dr. Volker Moll aus Aschaffenburg zwei Fälle: $30 \mathrm{mg}$ Dapoxetin, eine Stunde vor dem Geschlechtsverkehr verabreicht, verlängerte die IELT von weniger als zwei Minuten auf acht bis zwölf beziehungsweise sechs bis sieben Minuten, wie die Kontrolle nach drei Wochen zeigte. koc

Pressegespräch „Vorzeitiger Samenerguss in der Praxis. Mit Dapoxetin Sprachlosigkeit und Funktionsstörung überwinden“, Köln, 10. Februar 2010; Veranstalter: Janssen-Cilag

\section{Patienten-Ratgeber für Prostatakrebs}

- Das Prostatakarzinom die häufigste Tumorerkrankung des Mannes. Oft wird es mit einer Hormontherapie behandelt. Was aber, wenn es dann zum Progress kommt? Mit dem neuen Patientenratgeber möchte die Firma Sanofi- Aventis Patienten und Angehörige über die Behandlung des hormonrefraktären Prostatakarzinoms informieren. Neben medizinischen Hintergründen bietet die Wartezimmer-Broschüre Informationen über die Chemotherapie sowie Hilfestellung zum Umgang mit der Erkrankung und ein Glossar der wichtigsten Fachbegriffe. Ärzte und Kliniken können die Broschüre kostenlos über www.onkokolleg.de anfordern.

Nach Informationen von Sanofi-Aventis 